\title{
Références bibliographiques du dossier « Les espaces scolaires »
}

Hélène Beaucher

\section{(2) OpenEdition}

1 Journals

Édition électronique

URL : http://journals.openedition.org/ries/3635

DOI : 10.4000/ries.3635

ISSN : 2261-4265

Éditeur

Centre international d'études pédagogiques

Édition imprimée

Date de publication : 1 décembre 2013

Pagination : 163-173

ISBN : 978-2-85420-601-2

ISSN : 1254-4590

\section{Référence électronique}

Hélène Beaucher, "Références bibliographiques du dossier " Les espaces scolaires » », Revue

internationale d'éducation de Sèvres [En ligne], 64 I décembre 2013, mis en ligne le 01 décembre 2013,

consulté le 03 mai 2019. URL : http://journals.openedition.org/ries/3635; DOI : 10.4000/ries.3635

(c) Tous droits réservés 


\title{
Références \\ bibliographiques
}

\section{Hélène Beaucher}

Comment les espaces scolaires, lieux d'apprentissage et de socialisation sont-ils pensés? Comment peuvent-ils contribuer à la réussite des élèves ? Pour cette bibliographie non exhaustive et organisée en quatre parties, le choix s'est porté sur des publications pour la plupart très récentes. Des références liminaires apportent quelques éléments relatifs aux tendances éducatives dans les pays de l'OCDE, notamment en matière d'apprentissage. Cette première partie expose les enjeux et recense des documents qui privilégient une approche historiographique des espaces scolaires. La deuxième partie porte sur les nouveaux espaces scolaires. Nombre de changements, tels que les usages du numérique ou de nouvelles conceptions pédagogiques, réinterrogent aujourd'hui les espaces scolaires et l'architecture de l'école. Des études de cas illustrent les évolutions. Les impacts des espaces scolaires sont examinés dans la troisième partie. La dernière partie propose des études transnationales et nationales. Bibliographie arrêtée le 25 octobre 2013.

\section{ÉLÉmENTS de CADRAGE}

CERI : centre pour la recherche et l'innovation dans l'enseignement, OCDE, Les grandes mutations qui transforment l'éducation, OCDE/Paris, 2010, 94 p.

L'ouvrage donne un aperçu des principales tendances économiques, sociales, démographiques et technologiques et analyse leur impact sur l'éducation. Il a pour but d'aider les acteurs du domaine de l'éducation à réfléchir sur ces développements, autour de cinq grandes thématiques : les dynamiques de la mondialisation ; les nouveaux défis sociaux ; un monde du travail en pleine mutation; le nouveau visage de l'enfance; les TIC. Les auteurs distinguent une trentaine de tendances, regroupées selon ces cinq thématiques.

\section{DUMONT Hanna, BENAVIDES Francisco (éds), Comment apprend-on?} La recherche au service de la pratique, OCDE/Paris, 2010, 356 p.

Que savons-nous de la façon dont nous apprenons? Quelle influence les motivations et les émotions des jeunes ont-elles sur leurs apprentissages? Le travail en groupe, l'évaluation formative ou les pédagogies par projet donnent-ils de bons résultats? Qu'en est-il de l'apprentissage à l'aide des technologies ou au sein de la famille ? Les auteurs ont pour objectif d'établir un pont entre la recherche sur l'apprentissage des élèves et le monde des politiques publiques et des pratiques. Les contributions apportent des éclairages sur la nature de l'apprentissage et analysent les implications pour la conception de l'enseignement.

\section{Approches historiographiques}

\author{
CHÂTELET Anne-Marie, LE CEUR Marc (sous la direction de), "L'architecture \\ scolaire : essai d'historiographie internationale : dossier ", Histoire de l'éducation, \\ mai 2004, 306 p. \\ L'intérêt pour l'architecture scolaire ne s'est éveillé que progressivement. Compte-tenu de \\ l'importance des recherches aujourd'hui, ce numéro a pour objectif de dresser un bilan histo- \\ riographique international. Sans prétendre être exhaustif, il a pour ambition de présenter un \\ état des recherches, de comprendre l'évolution des travaux et de confronter leur développe- \\ ment suivant les pays retenus (Allemagne, États-Unis, France, Grèce, pays d'Afrique franco- \\ phone, Royaume-Uni, Suisse) et les disciplines.
}


CHÂTELET Anne-Marie, "Essai d'historiographie I: L'architecture des écoles au $X X^{e}$ siècle ", Histoire de l'éducation, $n^{\circ} 102$, mai 2004, p. 5-37

L'histoire de l'architecture des écoles primaires débute vers 1950. Elle est essentiellement écrite par des historiens de l'éducation et des historiens de l'architecture qui ont longtemps considéré les bâtiments scolaires comme un sujet marginal. Les premiers s'intéressaient aux théories pédagogiques, les seconds aux ouvres esthétiquement remarquables. Cet essai historiographique dégage les grands traits de cette histoire, en examinant cinquante ans de publications (1950-2000) et plusieurs pays, dont cinq dans lesquels l'enquête a été menée de façon systématique : l'Angleterre, les États-Unis, la Suisse, la France et l'Allemagne.

DEROUET-BESSON Marie-Claude, "L'apport de l'école à la construction d'une culture architecturale en France ", Revue de l'Inspection générale, $n^{\circ} 2$, février 2005, p. 4-19

Les questions foisonnent face aux multiples références pédagogiques ou éducatives qui sont évoquées lors des programmations de construction ou de rénovation des établissements scolaires. Quels repères dégager et quelles formes spatiales pour des établissements de qualité ? Selon l'auteure, il s'agit d'une entreprise difficile, voire impossible, en raison de la diversité des points de vue. L'article interroge dans la durée les constantes du cheminement du projet pédagogique au projet architectural en France et souligne que la généalogie des lieux d'école est pédagogique et éducative.

FORSTER Simone, "Architecture scolaire: un regard historique tourné vers l'avenir " in RHYN E., MICEL-ALDER E., STADELMANN W. (éds), Les enveloppes architecturales des lieux d'apprentissage de demain : rapport de congrès, CDIP : Conférence suisse des directeurs cantonaux de l'instruction publique/Berne, 2006, p. 44-52

L'auteure retrace l'histoire de l'architecture scolaire et des pédagogies en Suisse depuis le début de l'instruction obligatoire jusqu'à la transformation des écoles traditionnelles en écoles modulaires, en passant par le mouvement des écoles nouvelles, les écoles pavillonnaires et les écoles de plein air.

\section{LE CEUR Marc, "Essai d'historiographie II : Des collèges médiévaux aux campus ", Histoire de l'éducation, $n^{\circ}$ 102, mai 2004, p. 39-69}

L'intérêt des historiens pour le patrimoine architectural des établissements d'enseignement secondaire et supérieur est apparu au cours XIX ${ }^{\mathrm{e}}$ siècle. Le nombre et la finalité de leurs travaux n'ont cessé depuis de varier selon les époques et les pays. Aujourd'hui, on ne compte plus les monographies d'édifices, mais les études proprement typologiques sont encore trop rares. Se fondant sur de nombreux exemples, essentiellement européens et américains, cet article tente de rendre compte de la diversité des sujets et des approches. Il en ressort que la manière dont a été longtemps étudiée l'histoire de l'architecture scolaire procède du rapport qu'une population entretient avec les établissements eux-mêmes, parfois aussi de considérations très empiriques.

PANASSIER Catherine, PUGIN Valérie, L'école dans son rapport avec son environnement social et urbain, Millénaire 3, Centre Ressources Prospectives du Grand Lyon/Lyon, janvier 2008, 23 p. disponibles sur http//www.millenaire3.com [en ligne]

Dans un premier temps, cette synthèse propose un regard sur le rapport de l'école française à son environnement, à travers une rétrospective historique des grandes mutations qu'elle a connues. L'urbanisation, la massification scolaire, la décentralisation, l'évolution du monde du travail vont avoir un profond impact sur l'école et modifier sa relation à son environnement. Puis les auteures 
développent les questionnements actuels qui traversent l'école et les différentes postures, nouvelles ou résurgentes, quant à ses relations futures avec son environnement social et urbain.

\section{Enjeux}

BERGER Christian (éd.), "L'architecture scolaire : dossier », Bulletin CIIP, $n^{\circ} 15$, 2004, 36 p.

Selon quels principes construit-on les écoles au XXI ${ }^{\mathrm{e}}$ siècle ? Tient-on suffisamment compte des besoins matériels des élèves ? Est-il judicieux de considérer d'abord l'organisation scolaire comme utile voire nécessaire aux bonnes conditions d'apprentissage? Si oui, dans quelle mesure l'architecture scolaire a-t-elle une influence? Quels enseignements en tirer pour renverser la subordination des principes de construction scolaires aux nécessités de l'instruction et de l'éducation, définis par une politique de l'éducation et cadrés? Telles sont les questions abordées par ce numéro.

\section{MAZALTO Maurice (Sous la direction de), Architecture et réussite éducative,} Fabert/Paris, 2008, 192 p.

L'école de la République est, depuis toujours, l'objet d'attentions et de débats passionnés mais qui concernent rarement les espaces et les bâtiments. Pourtant, l'architecture scolaire n'est pas neutre et a une influence très forte sur la qualité d'un établissement. Né de la rencontre entre des architectes, des enseignants et des responsables de collectivités, cet ouvrage montre qu'il est nécessaire de réfléchir à l'influence de l'architecture sur les missions de l'école. Il fournit des pistes pour que les usagers participent à l'amélioration des espaces scolaires, dans le but de favoriser la réussite de tous les élèves. Une première partie, comprenant des repères historiques, présente les différents enjeux liés à la construction et à la rénovation d'un bâtiment scolaire. Les auteurs se penchent ensuite sur les points de vue des usagers. Une dernière partie porte sur le thème de l'appropriation de l'architecture scolaire en établissement.

MUSSET Marie, De l'architecture scolaire aux espaces d'apprentissage : au bonheur d'apprendre? Dossier d'actualité de la Veille et Analyses, mai 2012, $n^{\circ} 75,19$ p. disponibles sur http//ife.ens-lyon.fr [en ligne]

L'édifice scolaire revêt une fonction politique, sociale et pédagogique. Si la pierre semble défier le temps, la massification scolaire, le renouvellement de la pédagogie, les TICE - ainsi que la décentralisation en France - ont fait changer l'école et donc l'espace scolaire. Ce dossier s'intéresse aux interrogations les plus récentes - et partagées par la plupart des pays - concernant l'architecture scolaire et sa mutation en « espace d'apprentissage » associant une réflexion sur l'espace à l'intégration pédagogique des ressources électroniques et à la prise en compte de la vision holistique de l'apprentissage. L'auteure souligne l'intérêt de rechercher un langage commun entre usagers, architectes et maîtres d'ouvrage.

\section{NOUVELLES MODALITÉS D'APPRENTISSAGE, NOUVEAUX ESPACES SCOLAIRES}

BARRETT Peter, ZHANG Yufan. 2009, Optimal learning spaces : design implications for primary Schools : technical report, SCRI : Salford Centre for Research and Innovation, University/Salford, 2009, 45 p. disponibles sur http//usir.salford. ac.uk [en ligne]

S'appuyant sur les résultats de recherches internationales sur les meilleures pratiques, ce rapport fournit des repères afin d'améliorer la qualité des environnements d'apprentissage 
dans l'enseignement primaire au Royaume-Uni et propose un large éventail de possibilités de conception afin de repenser l'espace scolaire. Après une première partie énonçant les principes de conception des établissements, il met en lumière les différentes conséquences qui en découlent et expose les différents critères techniques (éclairage, acoustique, température, qualité de l'air) et pédagogiques à prendre en considération. L'accent est mis sur la création d'espaces adaptés aux besoins des élèves et qui favorisent leur bien-être.

JISC : Joint Information Systems Committee, How innovative technologies are influencing the design of physical learning spaces in the post-16 sector, University of Birmingham/Birmingham, 2005,154 p. disponibles sur http//www.jisc.ac.uk [en ligne]

S'appuyant sur une vaste revue de la littérature et sur une enquête menée dans des établissements d'enseignement en Grande-Bretagne, cette étude a pour objectif de mettre en relief les facteurs essentiels à prendre en compte par les décideurs à toutes les étapes d'un projet de construction ou de réhabilitation d'espaces d'apprentissage. L'organisation de tous les espaces d'apprentissage doit refléter une orientation vers un apprentissage centré sur l'élève, collaboratif et en groupe, éminemment personnalisé et flexible. Les apprenants doivent avoir accès à des services en ligne et être en communication avec des systèmes d'apprentissage à tout moment, en fonction de leurs besoins. Les projets de construction devront s'efforcer de rendre possible l'intégration des changements à venir.

\section{HEFCE : Education Funding Council for England, Designing spaces for effective} learning : a guide to $21^{\text {st }}$ century learning space design, HEFCE /Bristol, 2006, 36 p. disponibles sur http//www.jisc.ac.uk

Les environnements éducatifs d'aujourd'hui favorisent-ils le développement des diverses compétences nécessaires aux étudiants ? Cette publication du Conseil pour le financement du l'enseignement supérieur anglais vise à permettre une meilleure compréhension de ce qu'est l'architecture du XXe siècle et résume les points essentiels à prendre en considération dans un projet de réhabilitation ou de construction. Le rapport souligne la nécessité de tenir compte des spécificités pédagogiques et de leurs évolutions (méthodes d'apprentissage en groupe de taille diverse, place des TICE, etc.) et d'encourager la spécialisation.

MCEETYA : Ministerial Council on Education, Employment, Training and Youth Affairs, Learning spaces framework: learning in an online world, MCEETYA/ Carlton South, 2008, 23 p. disponibles sur le site http//www.mceecdya.edu.au

Les nouvelles technologies et leurs usages (ordinateurs et téléphones portables, Internet, jeux vidéo, chats, blogs, wikis, etc.) font partie intégrante de la vie des élèves. La pensée est moins linéaire et plus visuelle, les jeunes sont davantage multitâches. De nouvelles modalités d'apprentissage se font jour, induisant de nouvelles modalités d'enseignement. Publié par le ministère de l'éducation australien, ce guide propose une réflexion sur les nouvelles dynamiques pédagogiques et sur la prise en compte des TIC. Il fournit un cadre comprenant des principes d'organisation des espaces d'apprentissage. Flexibles, modulables, motivants, ceux-ci doivent faciliter la créativité, afin d'accompagner les apprenants. Selon les auteurs, il est nécessaire de changer la culture des écoles.

\section{NAIR Prakash, FIELDING Randall, LACKEY Jeffery, The language of school design : design patterns for $21^{\text {st }}$ century schools, 2009, 214 p.}

Cet ouvrage synthétise les travaux de la recherche sur l'apprentissage en se fondant sur les meilleures pratiques en matière de planification et de conception des écoles. Définissant un nouveau vocabulaire architectural, il propose 28 modèles différents d'espaces scolaires. Les auteurs déplorent la lenteur des évolutions de l'espace scolaire et insistent sur la nécessité de mieux tenir compte des innovations pédagogiques. Selon eux, les guides sont trop prescriptifs, 
tandis que les idées ne sont pas adaptées à la réalité du terrain. Les espaces d'apprentissage doivent s'adapter pour répondre aux nouveaux besoins de méthodes pédagogiques diverses. En outre, il convient de favoriser les interactions sociales.

OBLINGER Diana G. (Sous la direction de), Learning spaces, Educause/Washigton, 2006, 444 p. disponibles sur http//www.educause.edu [en ligne]

L'espace, qu'il soit physique ou virtuel, peut avoir un impact significatif sur l'apprentissage. Les besoins des apprenants ont changé. Les technologies de l'information ont ouvert de nouvelles perspectives, que ce soit en favorisant l'accès à des mondes virtuels ou en facilitant une plus grande interaction grâce à l'utilisation d'outils collaboratifs. Les différentes contributions portent sur la façon dont les attentes et besoins des apprenants influencent ces espaces et sur le rôle des nouvelles technologies. Les auteurs interrogent le rôle des nouvelles technologies ainsi que les principes et activités qui facilitent l'apprentissage, dans une perspective de création de nouveaux environnements. La deuxième partie de l'ouvrage propose de nombreuses études de cas.

PEARLMAN Bob, "Designing new learning environments " in $21^{\text {st }}$ century skills : rethinking how students learn, BELLANCA James A. (ed.), BRANDT Ron (ed.), Solution Tree/Bloomington, 2010, p. 116-147

L'auteur considère que le modèle traditionnel de la plupart des espaces scolaires issu de la révolution industrielle est désormais dépassé. En s'appuyant sur l'étude de bâtiments scolaires anglais et américains novateurs conçus pour l'apprentissage collectif, il souligne que la forme suit la fonction dans ces exemples innovants. Désormais l'engagement, la résolution de problèmes et la communication doivent être facilités par les environnements d'apprentissage.

RIGOLON Alessandro, "Les plans de construction des écoles européennes $d u$ $X X I^{e}$ siècle : présentation ", CELE Échanges, 2010, $n^{\circ}$ 3, 9 p.

Cet article propose une vision générale des différents types de bâtiments scolaires en Europe, basée sur une analyse des morphologies et des implantations spatiales. Malgré la diversité des constructions, les approches tendent à évoluer vers une nouvelle vision des environnements d'apprentissage qui rejoint les toutes dernières réflexions pédagogiques. La salle de classe traditionnelle n'est plus le seul espace d'apprentissage. Cela entraîne une plus grande flexibilité dans l'utilisation des espaces et conduit à repenser globalement l'implantation des bâtiments.

\section{Études de cas}

CANTIN Raymond, "La salle de classe du $21^{e}$ siècle ", Clic - bulletin collégial des technologies de l'information et des communications, $n^{\circ} 73,2010$, disponible sur http//www.clic.ntic.org [en ligne]

L'auteur présente deux projets particulièrement innovants qui favorisent l'apprentissage actif, interactif et collaboratif : le projet TEAL (Technology Enabled Active Learning), qui renvoie à une salle de classe innovatrice du Massachusetts Institute of Technology (MIT) à Boston; et le projet SCALE-UP de l'université de l'État de Caroline du Nord. Sur le plan spatial, l'environnement a été conçu pour faciliter l'interaction entre les équipes.

DE GREGORI Alessandro, Reimagining the classroom : opportunities to link recent advances in pedagogy to physical settings, Mc Graw-Hill Research Foundation/ New York, 2011, 16 p. disponibles sur http//mcgraw-hillresearchfoundation.org [en ligne]

Dans le contexte américain, l'auteur se penche sur l'adéquation des salles de classe aux pratiques pédagogiques et éducatives $\mathrm{du} \mathrm{XXI}^{\mathrm{e}}$ siècle issues du modèle constructiviste et 
davantage centrées sur l'apprenant. Il présente trois environnements physiques innovants qui ont en commun, en dépit de leurs différences contextuelles et pédagogiques, d'avoir été conçus afin de soutenir le modèle d'apprentissage de chaque école. Non seulement l'environnement physique doit contribuer à soutenir et améliorer l'enseignement et l'apprentissage, mais il doit en constituer une composante à part entière.

Department of education and early childhood development, Pedagogy and space : transforming learning through innovation, Department of education and early childhood development/Melbourne, 2009, 35 p. disponibles sur http//www.flemingtonps. vic.edu.au [en ligne]

Explorant les meilleures pratiques qui ont été adoptées en Australie par les écoles et les enseignants, cette étude fournit un cadre afin d'améliorer les résultats d'apprentissage des élèves. Les méthodes d'apprentissage se sont déplacées vers des modèles d'apprentissage plus centrés sur l'élève, et en équipe. Les auteurs soulignent que la conception des espaces d'apprentissage doit intégrer les TIC, considérés comme des éléments clés d'apprentissage et d'enseignement.

FISHER Kenn, "Environnements pédagogiques actifs facilités par la technologie: une évaluation ", CELE Échanges, $n^{\circ}$ 2010/7, 2010, 8 p.

L'avènement des nouvelles technologies a ouvert de remarquables possibilités aux modèles d'apprentissage mixtes du XXI ${ }^{\mathrm{e}}$ siècle et a sérieusement remis en question le modèle d'enseignement et d'apprentissage traditionnel de la salle de classe hérité de la révolution industrielle. Cet article se penche sur les raisons et les modalités de l'émergence des environnements pédagogiques actifs facilités par la technologie. Il examine leur capacité à améliorer l'enseignement et l'apprentissage en explorant trois établissements innovants, aux États-Unis et en Australie. Les évolutions brouillent la frontière entre ce qu'il convient traditionnellement de considérer comme "l'environnement pédagogique construit» et les technologies d'information et de communication qui sont au service de ces espaces.

ROBINSON Leigh, ROBINSON Taylor, "Architecture scolaire: des exemples à suivre en Australie ", CELE Échanges, $n^{\circ}$ 3, 2009, 6 p.

Selon les auteurs, les bâtiments scolaires contemporains construits dans la ville de Perth, en Australie, fournissent un aperçu de quelques modèles architecturaux exemplaires. Comportant les caractéristiques essentielles des environnements d'apprentissage efficaces, ils offrent des espaces stimulants compatibles avec différentes approches d'enseignement et d'apprentissage. Leur conception a tenu compte des exigences d'adaptabilité, de flexibilité, de la culture collective des élèves et des impératifs de durabilité.

\section{IMPACT DES ESPACES SCOLAIRES}

BARRETT Peter, ZHANG Yufan, MOFFAT Joanne, et al., "A holistic, multi-level analysis identifying the impact of classroom design on pupils' learning ", Building and Environment, janvier 2013, p. 678-689, disponible sur http//www.sciencedirect. com [en ligne]

Cet article rend compte d'une étude menée dans le but de repérer s'il existe des preuves tangibles de l'impact de la conception du bâtiment scolaire sur les résultats d'apprentissage des élèves. Prenant en compte les apports des neurosciences, l'étude a été élaborée à partir d'un échantillon d'élèves de l'enseignement primaire dans sept établissements scolaires au RoyaumeUni. Plusieurs paramètres liés à la conception de la salle de classe, tels que la couleur et la luminosité, influent sur les progrès d'un élève sur un an. Le plan de la classe, surtout s'il favorise les méthodes d'apprentissage et des activités variées (flexibilité), s'est révélé très important, de même que les caractéristiques qui favorisent le développement d'un sentiment d'appartenance chez les élèves. 
BLACKMORE Jill, BATEMAN Debra, LOUGHLIN Jill, et al., Research into the connection between built learning spaces and student outcomes: literature review, Education Policy and Research Division, Department of Education and Early Childhood Development/Melbourne, 2011, 62 p. disponibles sur http//www. education.vic.gov.au [en ligne]

L'étude porte sur les relations théoriques et empiriques effectuées entre les espaces d'apprentissage et les performances des élèves. L'objectif de cette revue de la littérature est de savoir si les nouveaux espaces d'apprentissage changent les pratiques d'enseignement de façon à améliorer les résultats d'apprentissage. Les auteurs abordent entre autres les questions de durabilité de l'environnement, de l'intégration des TIC, des partenariats avec le monde de l'industrie, de la revitalisation des quartiers ainsi que de la restructuration de l'organisation scolaire et de la gouvernance.

CUYVERS Katrien, DE WEERD Gio, DUPONT Sanne, et al., "Well-being a school: does infrastructure matter?", CELE Exchange, $n^{\circ} 10,2011,6$ p.

Quel est l'impact des infrastructures scolaires sur le bien-être des élèves dans les écoles secondaires flamandes ? Une étude, commandée par AGIOn, l'agence flamande qui subventionne les bâtiments scolaires, a étudié l'impact des espaces éducatifs sur leurs utilisateurs et a entrepris d'identifier des preuves empiriques à l'appui de l'importance des infrastructures scolaires sur le bien-être des élèves dans les écoles secondaires.

HIGGINS Steve, HALL Elaine, WALL Kate, et al., The impact of school environments : a literature review, Design Council/ London, 2005, 47 p. disponibles sur http//www.nl.ac.uk [en ligne]

S'appuyant sur une revue de la littérature, principalement anglaise et américaine, les auteurs interrogent les effets des environnements d'apprentissage sur la réussite des élèves, leur motivation, leur comportement et leur bien-être.

LIPPMAN Peter C., "L'environnement scolaire peut-il avoir un impact sur l'environnement pédagogique ", CELE Échanges, $n^{\circ} 13,2010,7$ p.

Cet article souligne la nécessité de remettre en question les «meilleures pratiques » généralement reconnues au sein de la profession architecturale et de créer des environnements pédagogiques en adoptant une approche basée sur l'architecture adaptée aux besoins des utilisateurs (responsive design). Celui-ci tient compte en particulier du rôle du contexte social et consiste à structurer l'environnement physique de façon à promouvoir l'apprentissage.

SCHNEIDER Mark, Do school facilities affect academic outcomes, NCEF : National Clearinghouse for Educational Facilities/Washington, 2002, 24 p. disponibles sur http//www.edfacilities.org [en ligne]

L'auteur s'intéresse aux caractéristiques de l'aménagement des espaces scolaires les plus susceptibles d'avoir une influence sur les résultats des élèves. Six critères ont été pris en compte : la qualité de l'air, la ventilation et la température, l'éclairage, l'acoustique, la qualité du bâtiment, la taille de l'école et celle de la classe.

ULINE Cynthia, TSCHANNEN-MORAN Megan, "The walls speak: the interplay of quality facilities, school climate, and student achievement ", Journal of Educational Administration, vol. $46 n^{\circ}$ 1, 2008, p. 55-73

Aux États-Unis, un nombre croissant de recherches explorant le lien entre la qualité des espaces scolaires et la réussite des élèves accompagne les efforts d'amélioration des infrastructures scolaires. En se basant sur une enquête menée dans des établissements secondaires, 
ce document s'intéresse à l'influence des infrastructures sur le climat scolaire et, par ricochet, sur les performances des élèves. Les résultats mettent en lumière une corrélation significative entre la qualité des installations scolaires et la réussite des élèves en anglais et en mathématiques.

\title{
Projet Éducatif ET CONSTRUCTION SCOLAIRE
}

\section{Études transnationales}

\begin{abstract}
DUDEK Mark, Écoles primaires et jardins d'enfants : manuel du projet, Infolio/ Gollion, 2007, 256 p. (Archigraphy)

Quelle relation y a-t-il entre vision pédagogique et construction scolaire ? Cette question est fondamentale pour comprendre les enjeux de l'architecture dans le milieu scolaire. Les théories pédagogiques évoluent sans cesse. La conception de l'environnement scolaire se transforme en conséquence. Après un rappel historique des systèmes éducatifs, ce guide présente aux architectes les principes de construction des écoles et jardins d'enfants : les fonctions, la configuration et la planification des espaces, l'acoustique, l'éclairage, la construction durable, la transformation des écoles et des jardins d'enfants en fonction de l'évolution de la pédagogie. Présentant plus de 80 projets en Europe, en Amérique et dans la région du Pacifique, il illustre les stratégies les plus récentes.
\end{abstract}

CELE : Centre for effective learning environments: "Standardised design" for schools. Old solution, new context?, OCDE/Paris, 2011, 12 p. disponibles sur http//www.oecd.org [en ligne]

À partir des éléments présentés lors d'une conférence internationale en ligne, ce rapport interroge le concept de standardisation et en présente les enjeux, implications et impacts pour la conception des bâtiments scolaires. Six exemples de mise en œuvre de cette approche en Australie, Belgique, Canada, Irlande, Mexique et Portugal sont présentés.

\section{OECD, Designing for Education: compendium of exemplary educational} facilities, OECD/Paris, 2011, 254 p.

Ce recueil propose une sélection de 60 établissements d'enseignement (écoles et universités) illustrant les meilleures pratiques en matière d'environnements pédagogiques efficaces. Ceux-ci doivent fournir un environnement qui s'adapte aux processus d'apprentissage et les améliore, encourager l'innovation, et répondre à la fois aux besoins actuels et aux demandes incertaines $\mathrm{du}$ futur. Les contributions de la première partie proposent une rétrospective des concepts développés depuis plus de 50 ans et une analyse des enjeux. Une deuxième partie présente des exemples d'établissements remarquables - écoles et universités - répartis sur six continents et sélectionnés par un jury international d'architectes.

\section{CELE : Centre pour des environnements pédagogiques efficaces, Environnements d'apprentissage du XXI siècle, OCDE/Paris, 2006, 106 p.}

Ce rapport rend compte des innovations qui caractérisent la conception des équipements éducatifs actuels, en présentant des études de cas qui concernent l'Australie, le Canada, la Nouvelle-Zélande, Singapour, la Suisse, le Royaume-Uni et les États-Unis. Les auteurs examinent les répercussions des nouvelles technologies, du confort des usagers et de l'implication des parties prenantes sur la conception des bâtiments. Équipements éducatifs conçus en tant qu'outils d'apprentissage, éducation permanente, inclusion, développement durable, durabilité, confort constituent autant de concepts qui sous-tendent les nouveaux espaces d'apprentissage. 


\section{Études nationales}

BLYTH Alastair, ALMEIDA Rodolfo, FORRESTER David, et al., The better schools programme, OCDE/Paris, 2012, 115 p.

Cet ouvrage présente une revue du programme mexicain mis en place en 2008 afin d'améliorer l'infrastructure physique des écoles pour l'éducation de base dans tout le pays. Une des caractéristiques clés du programme réside dans la participation sociale et l'engagement de chaque communauté scolaire.

\section{BLYTH Alastair, ALMEIDA Rodolfo, FORRESTER David, et al., Modernising secondary school buildings in Portugal, OCDE/Paris, 2012, 69 p.}

Ce rapport rend compte de l'évaluation par le CELE du programme national de rénovation et de construction des établissements d'enseignement secondaire au Portugal. Démarré en 2007, il est axé sur le confort environnemental et l'adéquation fonctionnelle des bâtiments à l'enseignement contemporain. Comment le programme permet-il de mieux répondre aux objectifs stratégiques du Portugal pour l'enseignement secondaire ? Quelle est la structure de sa gouvernance? L'évaluation s'intéresse à la qualité et la durabilité des bâtiments scolaires en vue de répondre aux besoins actuels et futurs, et à la manière dont les parties prenantes sont associées au processus.

CEPPI Guilo (ed.), ZINI Michele (ed.), Spaces, relations : metaproject or environment for young children, Grafiche Rebecchi Ceccarelli/Modena 1998, 160 p.

Cet ouvrage propose une analyse des écoles de Reggio Emilia, en Italie. Il formule des critères généraux de qualité et présente les caractéristiques souhaitables d'un espace pour les jeunes enfants. D'un point de vue organisationnel, une école reggiane est une école dont l'architecture a été travaillée et étudiée à des fins pédagogiques. Elle offre aux élèves des qualités spatiales qui prennent en compte leurs besoins, afin de stimuler leur goût pour l'apprentissage tout en leur proposant des espaces de jeu et d'expérimentation.

DARMODY Merike, SMYTH Emer, DOHERTY Cliona, Designing primary schools for the future, The Economic and social research institute/Dublin, 2010, 153 p. disponibles sur http//www.esri.ie [en ligne]

Se basant sur une enquête et six études de cas détaillées, les auteurs se penchent sur la conception des établissements d'enseignement primaire dans le contexte irlandais. L'étude souligne le rôle essentiel de la formation des enseignants à l'utilisation de l'espace pour le développement éducatif et social des élèves.

GRANOULHAC Françoise, "Construire l'école : de la planification à la privatisation, politiques publiques et architecture scolaire en Angleterre et au Pays de Galles depuis 1945 ", Revue LISA, 2006, disponible sur http//lisa.revues.org [en ligne]

Au cours des soixante dernières années, l'Angleterre a été à plusieurs reprises à l'avant-garde de la réflexion sur les lieux d'enseignement. L'auteure retrace l'évolution des politiques de construction scolaire de l'après-guerre à la période contemporaine, en tentant de dégager les points de rupture et les constantes et en mettant en lumière le rôle de l'État et des collectivités locales. Quelles ont été les conséquences des politiques sur la qualité technique et esthétique des bâtiments et sur la conception de l'espace scolaire? Les nouvelles dispositions permettent-elles de répondre durablement aux transformations du système éducatif et aux besoins des utilisateurs de l'école?

JETSONEN Sirkkaliisa, JOHANSSON Erika, The best school in the world: seven Finnish examples from the $21^{\text {th }}$ century, Museum of Finnish architecture/ Helsinki, 2011, 80 p.

Les auteurs étudient sept établissements scolaires finlandais et montrent que les architectes ont conçu les écoles en adéquation avec la philosophie éducative du pays, à savoir : un espace 
modulable, ouvert sur l'extérieur et confortable. Chacun de ces établissements répond à sa façon aux besoins des élèves et du corps enseignant. Les espaces extérieurs sont faciles à surveiller, les classes sont extrêmement modulables, de façon à modifier les espaces en fonction des besoins. Quel que soit le type d'activité, le lieu est capable de s'adapter simplement, tout en gardant un maximum de confort. L'école devient un lieu de vie essentiel pour les enfants, mais aussi pour la ville. Le bâtiment scolaire est le centre d'un rayonnement social et accueille les associations ou des ateliers le soir.

\section{LEEMANS Geert, VON AHLEFELD Hannah, Understanding school building} policy and practice in Belgium's Flemish Community, OECD Education working paper, $n^{\circ}$ 92, mai 2013, 29 p. disponibles sur http//www.oecd-ilibray.org/ [en ligne]

Ce document présente un cadre conceptuel pouvant être utilisé pour interpréter les questions de politique générale concernant la conception, la construction, l'entretien et l'évaluation des bâtiments scolaires de la Communauté flamande de Belgique. Il présente huit défis stratégiques concernant la mise à disposition des bâtiments scolaires en Flandre, susceptibles d'éclairer toute autorité nationale ou régionale s'intéressant aux thématiques relatives aux infrastructures scolaires. Le cadre conceptuel fait apparaître la construction scolaire comme un « nœud de relations ».

\section{LÜTHI Dave (sous la direction de), Lausanne : Les écoles, Société suisse d'histoire} de l'art/Berne, 2012, 256 p. (Architecture de poche)

Fruit d'un séminaire de recherche en l'histoire de l'art, cet ouvrage étudie une large palette d'édifices scolaires lausannois du début du XIX ${ }^{e}$ siècle aux années 1980. Les résultats des recherchesreplacent les édifices dans leur contexte historique en détaillant les enjeux de leur construction et de leur aménagement intérieur. Les réponses que les autorités ou les spécialistes apportent à ces questions sans cesse renouvelées témoignent tant de l'évolution de la pédagogie que de l'idéologie et des valeurs associées à l'école.

\section{France}

FEUILLATTRE Pauline, Une nouvelle architecture éducative pour les collèges de la Seine-Saint-Denis, Conseil général de la Seine-Saint-Denis/Bobigny, 2012, 54 p. disponibles sur http//seine-saint-denis.fr [en ligne]

Ce dossier présente les bases du programme de modernisation et de construction des collèges du département de Seine-Saint-Denis qui serviront de cadre de référence pour les futurs projets, un programme-type qui définit une nouvelle architecture éducative. Les orientations précises s'articulent autour des points suivants: le collège dans la ville, un cadre éducatif sécurisé, les parents partenaires, des espaces adaptés aux collégiens, la restauration scolaire, le centre de ressources au cœur du collège, un collège irrigué par les nouvelles technologies et respectueux de l'environnement; des espaces modulables au service de la pédagogie, et une pédagogie du patrimoine.

\section{MAZALTO Maurice, Cours de récréation et espaces de détente au collège et au lycée, Fabert/Paris, 2013, 143 p. (Les cahiers de l'architecture scolaire)}

Cet ouvrage questionne simultanément les temps et les espaces dédiés à la récréation et à la détente. Une enquête auprès de 800 collégiens et lycéens apporte des renseignements précieux sur leurs pratiques et leurs attentes. L'auteur relève une volonté commune de développer des lieux de rencontres et d'échanges qui favorisent le respect par la connaissance de l'autre et installent une vie sociale de qualité, facteur de réussite pour tous. 


\section{MAZALTO Maurice, L'accueil au collège et au lycée, Fabert/Paris, 2010, 100 p.}

Pendant très longtemps, l'accueil en établissement scolaire a été ignoré, en raison notamment d'une école renfermée sur elle-même, focalisée sur la transmission des savoirs. Mais les conceptions éducatives ont évolué et l'école reconnaît à présent l'importance de la socialisation dans ses murs ; l'accueil devient un facteur essentiel d'intégration et de bien-être. Comment l'architecture scolaire des collèges et lycées matérialise-t-elle cette évolution en France? Les lieux d'école étant d'une grande diversité, il n'existe pas de réponse unique. Néanmoins, des lignes de force, soulignées dans l'ouvrage, peuvent être dégagées.

Ministère de l'éducation nationale, de la jeunesse et de la vie associative, Vers des centres de connaissances et de culture, Ministère de l'éducation nationale, de la jeunesse et de la vie associative/Paris, mai 2012, 55 p. disponibles sur le site http// eduscol.education.fr [en ligne]

Dans les collèges et les lycées, les centres de documentation et d'information (CDI) évoluent progressivement pour devenir des espaces de ressources à la fois au centre des établissements scolaires et intégrés à leur environnement local. Certains établissements expérimentent le "centre de connaissances et de culture». À l'image des e-learning centres anglais, un centre de connaissances et de culture est un lieu d'accueil qui doit proposer aux élèves un cadre de travail et de rencontres convivial et chaleureux, pour faciliter l'implication des élèves. Il permet aussi de décloisonner espaces et temps scolaires, pour donner aux élèves plus d'autonomie dans leur parcours et plus d'occasions de collaborer entre eux. Ce vadémécum explore les principes et la démarche de cette expérimentation.

\section{SITOGRAPHIE}

\section{Best practices in educational facilities investments}

Conçu dans le cadre d'un projet sur la planification stratégique des investissements en infrastructures éducatives mené par le CELE et par la Banque européenne d'investissement, ce site fournit des informations sur la planification, la conception, la construction, la gestion et l'évaluation des espaces éducatifs. Il propose de nombreuses ressources pour des investissements stratégiques dans l'infrastructure éducative, dont une base de données qui recense des centaines de bonnes pratiques sous formes de fiches descriptives. http//edfacilitiesinvestment-db.org/

\section{CELE : Centre pour des environnements pédagogiques/OCDE}

Le CELE est un forum international unique rassemblant diverses perspectives et expériences pratiques pour traiter des questions de politiques éducatives relatives à l'environnement pédagogique. Il identifie et encourage les projets architecturaux centrés sur les utilisateurs, et satisfaisant à des critères de conception innovante, d'adéquation aux besoins, de durabilité et de sécurité. La mission du CELE est d'aider ses membres à tirer profit de la meilleure manière de leurs investissements dans les installations, les équipements éducatifs des écoles, des collèges des universités. Le site fournit de nombreuses ressources: rapports, recueils de bonnes pratiques, compte-rendu de projets, vidéos et conférences, ainsi qu'une revue : CELE Échanges. $\mathrm{http} /$ /www.oecd.org/fr/edu/innovation-education/centrepourdesenvironnementspedagogiquesefficacescele/

\section{NCEF : National Clearinghouse for Educational Facilities}

Le NCEF fait partie du programme du National Institute of Building Sciences, une organisation non gouvernementale officiellement reconnue comme référent américain pour la construction des espaces d'apprentissage du primaire au supérieur. Depuis 1998, le site propose des milliers de ressources pour les architectes, designers, politiques et enseignants. Tous les aspects de la construction scolaire y sont abordés : les questions de sécurité, de qualité environnementale ou d'esthétique. http//www.ncef.org/ 
